\title{
THE DESIGN OF TWO-DIMENSIONAL CONTRACTION SECTIONS*
}

By PAUL A. LIBBY AND HOWARD R. REISS (Polytechnic Institute of Brooklyn)

1. Introduction. The design of the expansion section of two-dimensional or axially symmetric supersonic nozzles has been the subject of considerable investigation. The methods to be used in their design are widely understood and readily applied. These are all based on the assumption of uniform, locally sonic flow at the minimum section. However, the literature on the design of a contraction section which will produce this uniform flow appears to be limited.

Tsien [1], Szczeniowski [2] and Smith and Wang [3] have presented methods for the design of axially symmetric contraction cones for an inviscid, incompressible fluid. There are, however, cases in which the contraction section is essentially two-dimensional and in which the inlet flow is approximately axial and uniform. For such contraction sections the authors were able to find no literature, although the above referenced methods could be applied to this case. However, the hodograph method [4] for incompressible, two-dimensional potential flow appears to offer a simpler solution to this problem.

2. Development. In this method the actual contraction section is taken from an infinite channel with asymptotes of $y= \pm \alpha$ at $x \rightarrow-\infty$ and of $y= \pm \beta$ at $x \rightarrow \infty$. The velocity at these extremes is in the $x$ direction and is equal to $a$ and $b$ respectively. It is required to find a contour connecting these asymptotes in such a fashion that the resultant velocity is everywhere monotonically increasing in the direction of flow. This requirement assures for both the incompressible and compressible flow that boundary layer separation and local compressibility effects will be avoided (cf. reference 1).

Although there may be chosen an infinite variety of functions, which give streamlines connecting points $(a, 0)$ and $(b, 0)$ in the hodograph $(u, v)$ plane and which satisfy this requirement, a simple set may be found as follows: Consider the complex potential $F=\phi+i \psi$

$$
F(\bar{w})=c[\ln (\bar{w}-b)-\ln (\bar{w}-a)],
$$

where $\bar{w}$ is the usual complex conjugate velocity, $u-i v$, and where $c$ is a positive, real constant. Considering the real and imaginary parts of Eq. (1), one finds that

$$
\begin{aligned}
& \phi / c=(1 / 2) \ln \left\{\left[(u-a)^{2}+v^{2}\right] /\left[(u-b)^{2}+v^{2}\right]\right\}, \\
& \psi / c=\tan ^{-1}[-v /(u-a)]-\tan ^{-1}[-v /(u-b)] .
\end{aligned}
$$

It is of interest to examine the streamlines in the $\bar{w}$ plane. From Eq. (2b) one obtains the equation

$$
\psi / c=\theta_{a}-\theta_{b}=\gamma,
$$

where these angles are shown in Fig. 1 . An examination of this figure indicates that the streamlines are arcs of circles passing through the points $(a, 0)$ and $(b, 0)$. Since the velocity at any point on such a streamline is represented in the $\bar{w}$ plane by a vector to that point from the origin, only those streamlines corresponding to $\pi / 2 \leq|\psi / c| \leq \pi$ will yield wall shapes in the $z$ plane with monotonically increasing velocities. The arcs

*Received June 5, 1950. 
corresponding to $|\psi / c|<\pi / 2$ yield, in the $z$ plane, streamlines along which the velocity is locally decreasing in two regions. Figure 1 also indicates that the centerline of sym-

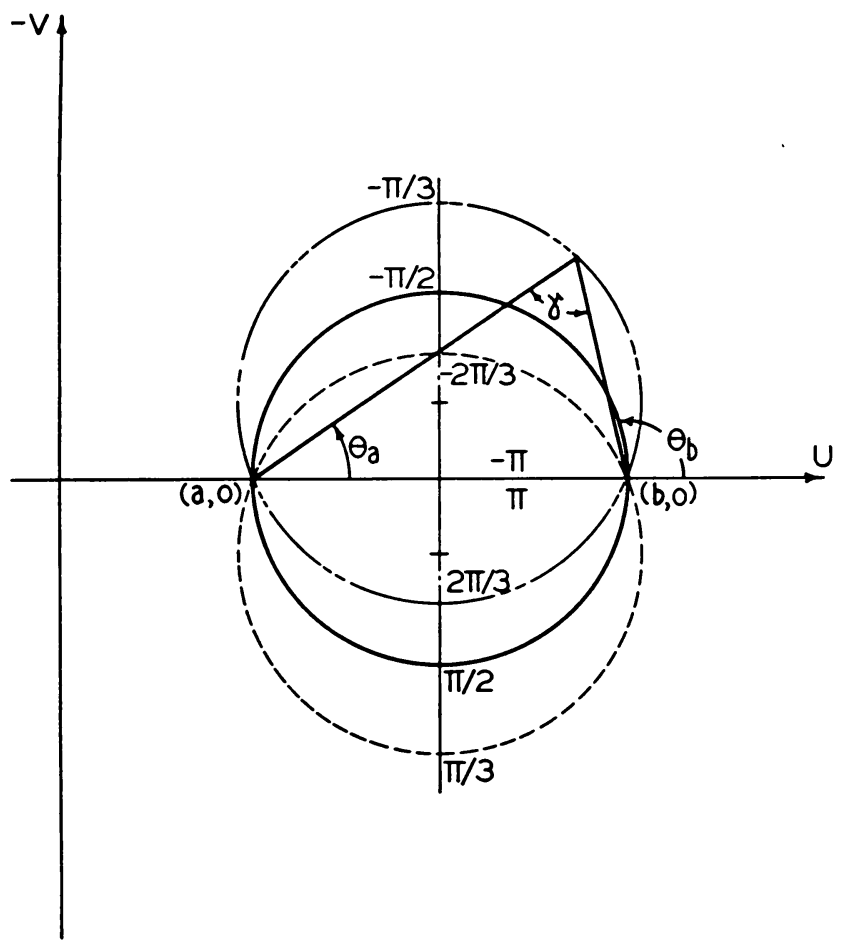

Fig. 1. Streamlines in the $\bar{w}$ plane.

metry of the contraction section corresponds to $\psi / c= \pm \pi$. The $x$ and $y$ coordinates of the streamlines will now be determined.

Solving for $\bar{w}$ from Eq. (1), one obtains

$$
\bar{w}=[b-a \exp (-F / c)] /[1-\exp (-F / c)] .
$$

From the definition of $F$, the complex variable $z=x+i y$ is given by the transformation equation

$$
z=\int(\bar{w})^{-1} d F+C_{0}
$$

or from Eq. (3)

$$
z=\int\{[1-\exp (-F / c)] /[b-a \exp (-F / c)]\} d F+C_{0},
$$

which, upon integration gives

$$
z=(F / b)-(c / b)[(b / a)-1] \ln [(b / a)-\exp (-F / c)]+C_{0}^{\prime},
$$

where $C_{0}$ and $C_{0}^{\prime}$ are arbitrary complex constants of integration. 
Now with $F=\phi+i \psi$ one obtains, by equating the real and imaginary parts of each side of Eq. (6),

$$
\begin{gathered}
x=(\phi / b)-(c / b)[(b / a)-1] \ln \left\{1+[a \exp (-\phi / c) / b]^{2}\right. \\
-[2 a \cos (\psi / c) \exp (-\phi / c) / b]\}^{1 / 2}+C_{1}, \\
y=(\psi / b)-(c / b)[(b / a)-1] \\
\cdot \tan ^{-1}\{[\exp (-\phi / c) \sin (\psi / c)] /[(b / a)-\exp (-\phi / c) \cos \psi / c]\}+C_{2},
\end{gathered}
$$

where again $C_{1}$ and $C_{2}$ are arbitrary real constants. To obtain the streamlines of the flow given by Eqs. (7a) and (7b) one may consider them, for $\psi=$ constant, two parametric equations giving $x$ and $y$ in terms of $\phi$.

To determine $c$ and $C_{2}$, Eqs. (7a) and (7b) are examined. For $\phi \rightarrow-\infty, x \rightarrow-\infty$ provided $b / a>1$, and

$$
y \rightarrow(\psi / b)-(c / b)[(b / a)-1][ \pm \pi-(\psi / c)]+C_{2},
$$

where the upper and lower signs correspond to $\psi / c \gtrless 0$ respectively. Furthermore, for $\phi \rightarrow \infty, x \rightarrow \infty$,

$$
y \rightarrow(\psi / b)+C_{2},
$$

where the arctangent is chosen in the first and second quadrant for $\psi / c>0$ and in the third and fourth quadrant for $\psi / c<0$.

The constant $C_{2}$ is determined so that $\psi / c= \pm \pi$ along the $x$ axis $(y=0)$. Thus from Eq. (7b)

$$
C_{2}=\mp c \pi / b,
$$

giving as

$$
\phi \rightarrow-\infty, \quad y \rightarrow[(\psi / c) \mp \pi] /(a / c),
$$

and as

$$
\phi \rightarrow \infty, \quad y \rightarrow[(\psi / c) \mp \pi] /(b / c) .
$$

Now if it is required that as $x \rightarrow-\infty(\phi \rightarrow-\infty), y \rightarrow \mp \alpha$ for $\psi / c= \pm \psi_{0}$, i.e., that the contraction section walls correspond to $\psi / c= \pm \psi_{0}$, then from Eq. (9b)

$$
\alpha=\left(-\psi_{0}+\pi\right) /(a / c)
$$

or

$$
c=a \alpha /\left(-\psi_{0}+\pi\right) \text {. }
$$

Then as $x \rightarrow \infty(\phi \rightarrow \infty)$, from Eqs. (9c) and (10a) it follows that

$$
y \rightarrow \mp \alpha \alpha / b \equiv \mp \beta .
$$

Thus if $\Phi \equiv(a / b) \exp (-\phi / c)$, Eqs. (7a) and (7b) with Eqs. (9a), (10a) and (10b) yield for the wall contour in the upper half of the $z$ plane $\left(\psi / c=-\psi_{0}\right)$ denoted by the subscript zero

$$
\begin{aligned}
\xi_{0}= & \left(x_{0} / \alpha\right) \\
& =-\left[(\beta / \alpha) /\left(-\psi_{0}+\pi\right)\right]\left\{\ln \Phi+1 / 2[(\alpha / \beta)-1] \ln \left(1+\Phi^{2}-2 \Phi \cos \psi_{0}\right)\right\}
\end{aligned}
$$


and

$$
\begin{aligned}
\eta_{0}= & \left(y_{0} / \alpha\right)=\left[(\beta / \alpha) /\left(-\psi_{0}+\pi\right)\right] \\
& \cdot\left\{-\psi_{0}-[(\alpha / \beta)-1] \tan ^{-1}\left[-\Phi \sin \psi_{0} /\left(1-\Phi \cos \psi_{0}\right)\right]+\pi\right\} .
\end{aligned}
$$

The arbitrary constant $C_{1}$ in Eq. (7a) leads only to a translation of the streamlines along the $x$ or $\xi$ axis and thus may conveniently be set equal to zero.

Equations (11a) and (11b) give the final contour in the upper half plane in terms of the parameter $\Phi(0 \leq \Phi<\infty)$. Note that $\xi_{0} \rightarrow-\infty$ and $\eta_{0} \rightarrow 1$ as $\Phi \rightarrow \infty$, and $\xi_{0} \rightarrow \infty$ and $\eta_{0} \rightarrow \beta / \alpha$ as $\Phi \rightarrow 0$. In order to obtain the streamlines inside the wall contour, $\psi / c$ may be permitted to vary from its wall value, $-\psi_{0}$, to $-\pi$. It might be pointed out that for asymmetrical channels $\psi_{0}$ would simply take on different values in the upper and lower halves of the $z$ plane.

3. Numerical Example. In Fig. 2 the wall contours for $\alpha / \beta=4$ with $\psi_{0}=\pi / 2$, $2 \pi / 3$ and $\pi / 3$, are shown. Note that the latter contour, for which the velocity is not
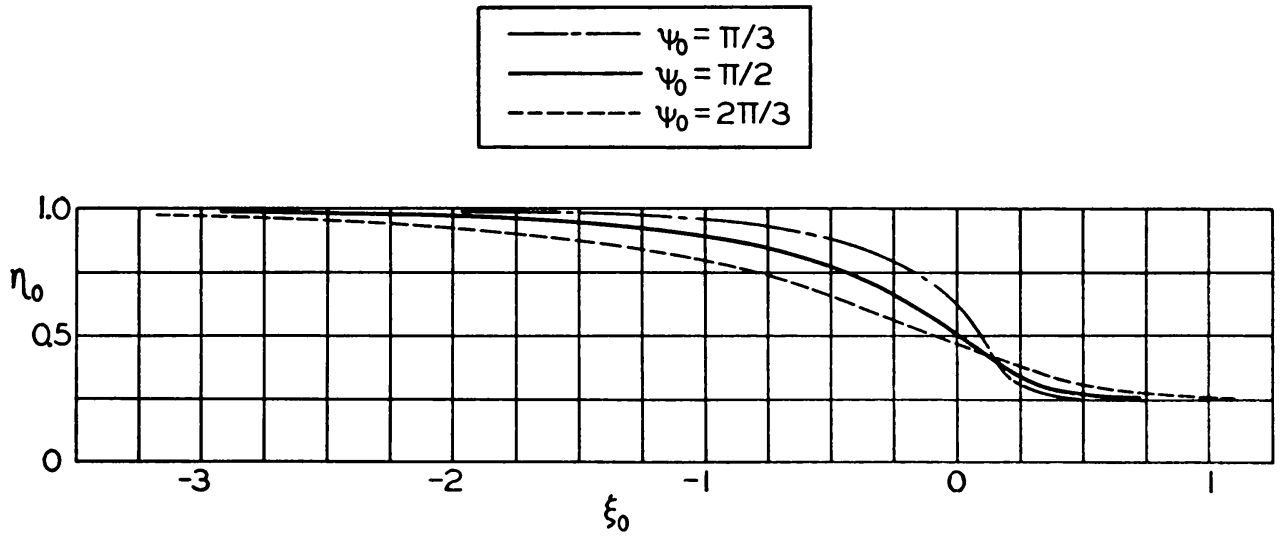

Fig. 2. Wall contours. Contraction ratio $=4$.

monotonically increasing, appears to be reasonable and might well be drawn and selected for a contraction cone if no analysis were carried out. It will be noted that the asymptotic values, 1 and $\beta / \alpha$ are approached quickly. Criteria of closeness to the asymptotes, of uniformity of the $u$ velocity, or of the smallness of the $v$ velocity can be applied to establish the values of $\xi_{0}$ determining the length of the section.

\section{REFERENCES}

1. H. S. Tsien, On the design of the contraction cone for a wind tunnel, J. Aero. Sciences 10, 68-70 (1943).

2. B. Szczeniowski, Contraction cone for a wind tunnel, J. Aero. Sciences 10, 311-312 (1943).

3. R. H. Smith and C. T. Wang, Contracting cones giving uniform throat speeds, J. Aero. Sciences 11, 356360 (1944).

4. L. Prandtl and O. G. Tietjens, Fundamentals of hydro- and aeromechanics, McGraw-Hill, 1934, pp. 178-183. 